\title{
BARRIER TREATMENTS USING COMBINED LAMBDA-CYHALOTHRIN AND PYRIPROXYFEN REDUCE PERIDOMESTIC AEDES MOSQUITOES IN A SUBTROPICAL ENVIRONMENT
}

\author{
ROBERTO M. PEREIRA', CARRIE COTTONE², CLAUDIA RIEGEL², \\ CHRIS KEEFER ${ }^{3}$, MOHAMED F. SALLAM ${ }^{4 *}$ AND PHILIP G. KOEHLER ${ }^{1}$ \\ University of Florida, Department of Entomology \& Nematology, \\ Gainesville, FL
}

${ }^{2}$ New Orleans Mosquito and Termite Control Board, New Orleans, LA

${ }^{3}$ Syngenta Crop Protection, Inc., Greensboro, NC

${ }^{4}$ Biology Department, College of Science, University of Nevada-Reno, Reno, NV

*Corresponding author: msallam@unr.edu

Subiect Editor: Seth Britch

\begin{abstract}
Barrier treatment of vegetation using lambda-cyhalothrin has been shown to be effective at reducing adult mosquito populations in the US. However, recent investigations have indicated that standard residual adulticide barrier treatments may be enhanced when combined with an insect growth regulator targeting immature stages that could be transferred to immature habitat by adults contacting treated surfaces. We conducted field trials at residential sites in a subtropical urban environment in north central Florida treating blocks of vegetation with residual sprays of lambda-cyhalothrin (Demand ${ }^{\circledR} \mathrm{CS}$ ) and pyriproxyfen (Archer®) alone and in combination treatments to determine their efficacy against peridomestic mosquitoes. The combined treatment resulted in consistent approximately $100 \%$ reduction in Aedes mosquito eggs for $16 \mathrm{wk}$ post-treatment compared to not significantly lower but more variable reductions at alone treatment sites.
\end{abstract}

Key Words: barrier treatment; disease vectors; insect-growth regulator; pyrethroid; residual adulticide

Peridomestic barrier treatments to surrounding vegetation for control of adult disease vector mosquitoes have shown efficacy of $85-100 \%$ reduction in collection of adult mosquitoes in urban settings and could be considered a proven method for urban mosquito control (Trout et al. 2007, Unlu et al. 2018, Stoops et al. 2019). Recently, however, studies have been conducted in New Jersey in the northeastern US to enhance this control technique by combining an insect growth regulator with the residual adulticide that could be transferred to immature habitat by females that contacted the treated barrier and survived long enough to oviposit (Unlu et al. 2018, Williams et al. 2019). In this study we investigated whether this enhanced barrier treatment technique could be effective in a warm subtropical habitat in the southeastern US in north central Florida.

We conducted field applications to evaluate the residual efficacy of lambdacyhalothrin (Demand® CS; Syngenta Crop Protection, Inc., Greensboro, NC) and pyriproxyfen (Archer®; Syngenta Crop Protection, Inc., Greensboro, NC) against peridomestic mosquitoes as a barrier treatment in nine approximately 1 A residential sites with dense foliage perimeters on the University of Florida campus in Gainesville, FL. Three sites were randomly selected for treatment with $0.06 \%$ lambda-cyhalothrin alone, three 
for combined treatment with $0.06 \%$ lambdacyhalothrin $+0.01 \%$ pyriproxyfen, and three for untreated controls. Limited resources precluded investigation of $0.01 \%$ pyriproxyfen on its own. We used a Stihl® SR 200 (STIHL Inc., Virginia Beach, VA) backpack mist blower on the number three setting for all barrier spray treatments, with a target application rate of $0.61 \mathrm{~L} / \mathrm{min}$. All field trials were conducted during July 5-October 18, 2016.

We measured efficacy of the barrier treatments by deploying five $450 \mathrm{~mL}$ ovicups (Creative Converting Inc., Clintonville, WI) filled with $150 \mathrm{~mL}$ of tap water and a $15 \mathrm{~cm}$ $\mathrm{x} 2 \mathrm{~cm}$ wooden tongue depressor (Fisherbrand, Pittsburgh, PA) during pre- and posttreatment periods in each study site to assess Aedes mosquito egg density. The tongue depressors were collected and replaced every $7 \mathrm{~d}$ and returned to the laboratory for egg counts. The ovicups for the pre-treatment period were deployed two weeks before spray applications to establish the baseline density of Aedes eggs expected in the area from the natural populations. We applied two rounds of barrier treatment applications on the vegetation bordering each of the 6 treated sites: the first applied two weeks after collection of pre-treatment data, and the second applied eight weeks after the first treatment.

Efficacies of treatments were evaluated by egg counts at twelve intervals consisting of $1-8,10,12,14$, and 16 wk after the initial application. We downloaded publicly available weather data throughout field trials from the weather recorder at the Gainesville, FL regional airport approximately $8 \mathrm{~km}$ from study area. Mean egg densities were calculated for all sites for each sample period to determine the variance in egg density within each treatment - i.e., lambda-cyhalothrin, lambda-cyhalothrin + pyriproxyfen, and untreated control - over time and evaluate differences using a three-way $t$-test at the $P<$ 0.05 level using JMP version 12 (Boblingen, Germany).

The distributions of egg collection data for all sites over the 2 wk pre-treatment period are shown in Fig. 1, and the three-way $t$ test confirmed there were no significant differences among the mean number of Aedes mosquito eggs across the 9 sites designated for alone and combined treatments and untreated control (Table 1). Regarding post-

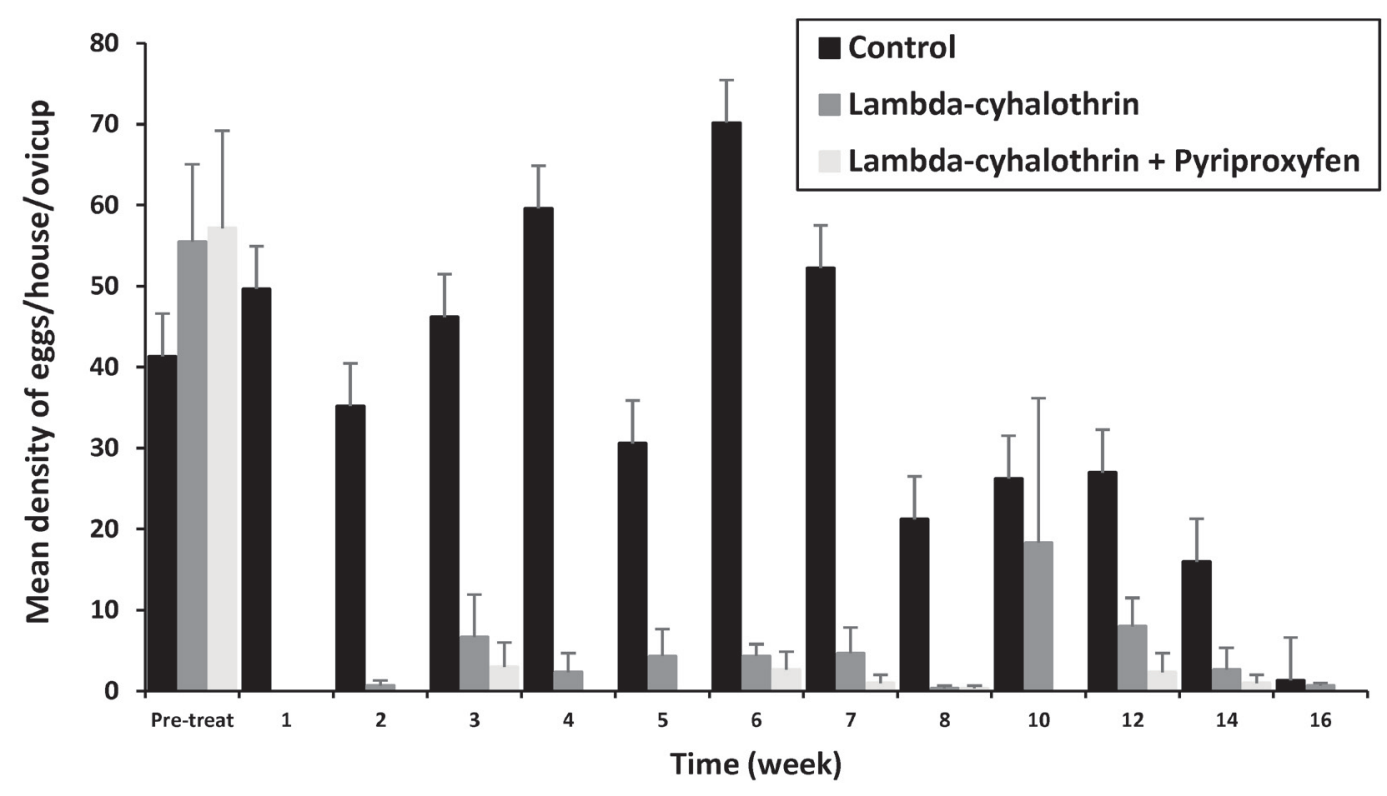

Figure 1. Mean number (standard error bars) of Aedes eggs collected by ovitraps per site (5 per site) for $2 \mathrm{wk}$ pre-treatment and for $16 \mathrm{wk}$ following alone and combination residual barrier treatments. The alone treatment consisted of $0.06 \%$ lambda-cyhalothrin, and the combined treatment consisted of $0.06 \%$ lambda-cyhalothrin + $0.01 \%$ pyriproxyfen. 
Table 1. The number of Aedes mosquito eggs (mean $\pm \mathrm{SE}$ ) collected from 9 study sites in Gainesville, FL for 2 wk before and $16 \mathrm{wk}$ after application of alone and combined barrier treatments.

\begin{tabular}{lcc}
\hline \hline Treatment (3 sites each) & $\begin{array}{c}\text { Pre-treatment } \\
\text { Mean } \pm \text { SE }\end{array}$ & $\begin{array}{c}\text { Post-treatment } \\
\text { Mean } \pm \text { SE }\end{array}$ \\
\hline lambda-cyhalothrin $0.06 \%$ & $55.5 \pm 9.57^{\mathrm{a}}$ & $4.3 \pm 1.59^{\mathrm{a}}$ \\
lambda-cyhalothrin $0.06 \%+$ pyriproxyfen $0.01 \%$ & $57.2 \pm 12.02^{\mathrm{a}}$ & $0.9 \pm 0.30^{\mathrm{a}}$ \\
Untreated Control & $41.3 \pm 31.8^{\mathrm{a}}$ & $38.6 \pm 7.61^{\mathrm{b}}$ \\
\hline
\end{tabular}

Means followed by the same letter in the same column are not significantly different $(P>0.05)$.

treatment egg collection data, there was an approximately $100 \%$ reduction in oviposition by Aedes mosquitoes at sites treated with the combined lambda-cyhalothrin and pyriproxyfen treatment for up to $16 \mathrm{wk}$ (Fig. 2). For the alone treatment with lambda-cyhalothrin, we observed a reduced (but non-significant) efficacy compared to the combined treatment sites with a mean value of 4.3 Aedes eggs post-treatment (Table 1). This non-significant overall reduction in efficacy shown in Table 1 between the combined and alone treatments is likely due to the reduced percent reductions compared to controls shown by the alone treatment in collections from weeks 10, 12, and 16 in Fig. 2. One explanation for this is that significantly elevated rainfall of $202 \mathrm{~mm}$ took place in the study area in September 2016, corresponding to egg collections at weeks 10 and 12, compared to monthly rainfall of $38-83 \mathrm{~mm}$ for July-August. Similarly this heavy rain event appeared to slightly decrease the impact of the combined residual barrier treatment at the $12 \mathrm{wk}$ and $14 \mathrm{wk}$ collections when compared side by side with controls (Fig. 1) and as a percent reduction compared to controls for the same collection speriods in Fig. 2.

Our results indicate that the combination of lambda-cyhalothrin and pyriproxyfen in a peridomestic residual barrier treatment enhanced the treatment in two ways compared to lambda-cyhalothrin on its own. First, the combined treatment resulted in consistently,

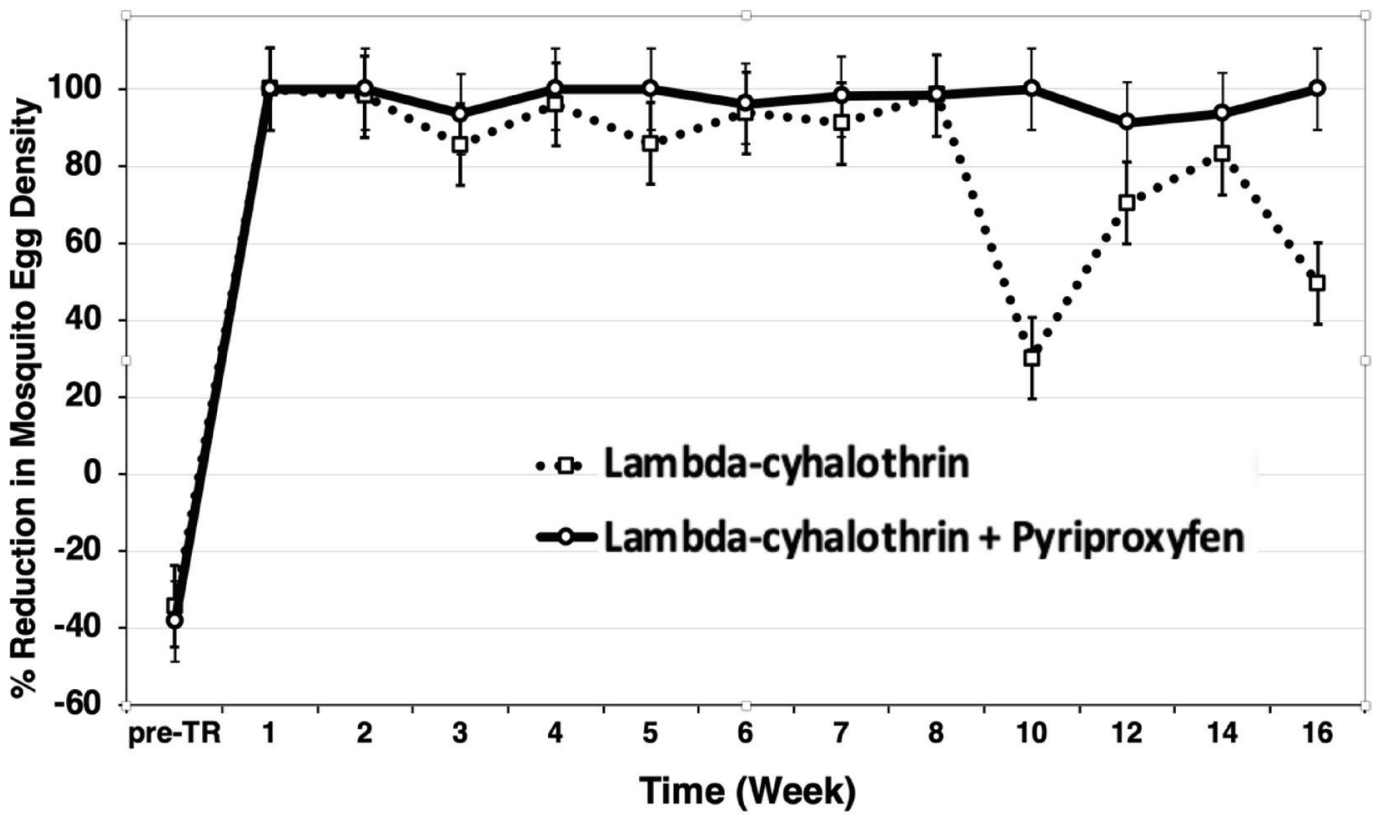

Figure 2. Percent reduction over 2 wk pre-treatment and 16 wk post-treatment in mean Aedes mosquito egg density collected at alone $0.06 \%$ lambda-cyhalothrin and combined $0.06 \%$ lambda-cyhalothrin $+0.01 \%$ pyriproxyfen residual barrier treatment sites compared to untreated control sites. 
though not significantly, greater reduction in egg collections throughout the study period. Second, the combined treatment provided more consistent performance through elevated rainfall conditions compared to the traditional barrier treatment on its own.

We hypothesize that in our investigation the combined treatment provided an integrated impact on adult and immature life stages that caused greater (though not significantly) and more consistent reductions in mosquito populations compared to alone treatments. Lambda-cyhalothrin is a synthetic pyrethroid adulticide and causes rapid knockdown effect to susceptible adult population of mosquitoes; pyriproxyfen indirectly reduces the number of adult mosquitos by slowing down the development of mosquito immatures and lead to failure in adult emergence. The combination of the larvicidal and adulticidal products theoretically increased the efficacy of the application by preventing the development of immature stages and survival of adult mosquitoes within the treated areas, and limiting survival of mosquitoes flying in from nearby untreated areas.

Future studies should include sites treated with a residual barrier treatment of pyriproxyfen on its own to tease apart the relative contributions of each active ingredient in the combined treatments. In addition, testing of the water in ovicups could detect whether pyriproxyfen is present, which could help determine the relative contributions of the adulticide and the insect growth regulator to reductions in egg collections. Future studies should also simultaneously collect adult Aedes mosquitoes in addition to egg collections across the study area to better evaluate whether residents could expect enhanced reductions in biting pressure from combine treatments. Finally, further investigation should be focused on whether the combined treatments can create a more weather-resistant integrated system that provides stable long term control.

\section{ACKNOWLEDGMENT}

This is a research report only and the mention of commercial products is to provide specific information and does not imply endorsement by University of Florida, New Orleans Mosquito and Termite Control Board, or University of Nevada-Reno. The research reported in this publication was supported by Syngenta; however, the content is solely the responsibility of the authors and does not necessarily represent the official views of Syngenta.

\section{REFERENCES CITED}

Stoops CA, Qualls WA, Nguyen TT, Richards SL. 2019. A review of studies evaluating insecticide barrier treatments for mosquito control from 1944 to 2018. Environ Health Insights. doi 10.1177/1178630219859004.

Trout RT, Brown GC, Potter MF, Hubbard JL. 2007. Efficacy of two pyrethroid insecticides applied as barrier treatments for managing mosquito (Diptera: Culicidae) populations in suburban residential properties. JMed Entomol 44:470-477.

Unlu I, Williams GM, Rochlin I, Suman D, Wang Y, Chandel K, Gaugler R. 2018. Effectiveness of lamdacyhalothrin and pyriproxyfen barrier treatments for Aedes albopictus (Diptera: Culicidae) management in urbanized areas of New Jersey. J Med Entomol 55:472476.

Williams GM, Unlu I, Rochlin I, Wang Y, Gaugler R. 2019. Timing of lambda-cyhalothrin and pyriproxyfen barrier treatments for Aedes albopictus (Diptera: Culicidae) management. J Econ Entomol 112:13371342. 